\title{
Blood in Saliva of HIV Seropositive Drug Abusers: Possible Implication in AIDS Transmission
}

\section{Ange Bissanga*‡}

\author{
Address: L'Association Soliderite Sainte, Congo \\ Email: Ange Bissanga* - solidaritesante1@yahoo.fr \\ * Corresponding author $\ddagger$ Presenting author
}

from 2005 International Meeting of The Institute of Human Virology

Baltimore, USA, 29 August - 2 September 2005

Published: 8 December 2005

Retrovirology 2005, 2(SuppI I):PI8 doi:I0.II86/1742-4690-2-SI-PI8

We have studied hemoglobin concentration in saliva of anti-HIV positive and anti-HIV negative intravenous drug abusers (IVDA) and normal controls and the relationship between hemoglobin concentration in saliva and number of CD4+ cells and clinical status of AIDS in anti-HIV positive IVDA. 120 anti-HIV positive IVDA' 112 anti-HIV negative IVDA and 116 normal healthy subJects not belonging to any risk group for HIV infection completed the study. Saliva was collected at awakening before brushing teeth and the concentration of hemoglobin was determined. Hemoglobin concentration in saliva in basal conditions is higher in anti-HIV positive IVDA with respect to anti-HIV negative IVDA ( $p$ less than 0.05) and controls ( $p$ less than 0.01). In anti-HIV positive IVDA hemoglobin concentration in saliva is higher in subjects with CD4+ cells less than 200/10(6) l with respect to subjects with CD4+ greater than 200/10(6) l (p less than 0.05 ) and in subjects with ARC/AIDS with respect to subjects with PGL or who are asymptomatic (p less than 0.01). SubJects with ARC/AIDS have a mean concentration of hemoglobin of 19 micrograms $/ 0.1 \mathrm{ml}$ saliva (range 0-153) which corresponds to 1.3 microliters of blood/ml saliva. If $10 \mathrm{ml}$ of saliva are exchanged during kissing an average of 13 microliters of blood are transferred (110 microliters of whole blood at extreme range). Blood of symptomatic patients has an HIV titer of 7 TCID/ microliters which for $10 \mathrm{ml}$ saliva containing an average of $1.3 \mathrm{microliters}$ blood $/ \mathrm{ml}$ saliva corresponds to an average of 90 TCID (770 TCID at the extreme range). 